\title{
Why the title of your paper matters
}

Titles are the first, and often the only, part of your paper that others will read. That's why they matter so much, and here's some practical advice on how to write them.

W hat is the most important part of a scientific paper? Is it the methods section, the results, the figures, or the raw data? All of these are crucial elements of any scientific work, but from the perspective of the average reader, the single most important part of a paper may be the title.

The title is by far the most-read part of a paper. A busy scientist scanning a list of new articles is unlikely to click beyond the title of most of them to access the full text. For the majority of readers, the title is the message of the paper. This is why it's so important that titles be succinct, accurate, and balanced in their presentation of the results.

When readers do decide to read the full text of an article, it is generally because they were interested by the title. A title serves to help direct attention to the paper. The best way to get people interested in your work is by choosing a title that clearly and straightforwardly describes what the paper is about and what it shows. Titles do their jobs best when they are simple, readable, and brief.

Humour is not something that titles need. Yes, when done well, joke titles can be funny-such as the famous "You probably think this paper's about you: narcissists' perceptions of their personality and reputation" or "Pressures produced when penguins pooh-calculations on avian defaecation". But witty titles are frequently culturally specific or rely on references to current events that will age poorly. "Fantastic yeasts and where to find them: the hidden

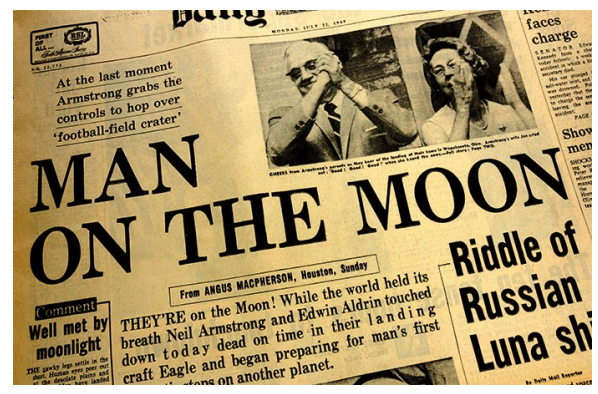

Credit: Alistair Scott / Alamy Stock Photo

diversity of dimorphic fungal pathogens" is a brilliant play on the title of a 2016 Harry Potter movie, but not every reader will be familiar with it, and people of the future are unlikely to appreciate the reference.

Even when a title does succeed in being hilarious, it may not serve its purpose, which is to tell people (and search engines) what your paper is about. Studies have suggested that funny titles don't translate to higher citations ${ }^{1}$ and may even result in fewer citations than papers with more matter-of-fact titles ${ }^{2}$.

Many journals, including Nature Human Behaviour, also ask authors to avoid question-based titles, such as "Is X involved in Y?" Although questions in titles let readers know what the topic of your paper is, they don't provide a take-home message. No matter what the answer to your question is-'yes', 'no', or 'it's complex'-readers are best served by knowing it from the outset.
So how can one write a great title? A title should simply be a summary of the most important findings of the paper. The best titles contain the essence of the paper, presented as clearly as possible.

If you're struggling to identify the essence of your work, imagine that your paper is a plot point in a movie. Many movies feature scenes where one character enters a room waving an important document or file in their hand. What's in the file? The character tells everyone, summarizing it in a single sentence. Thanks to the summary, the audience now knows what it means for the story. A title should be just like that kind of summary.

Make sure your title contains keywords to help search engines surface your paper in literature searches by colleagues working on similar questions. At the same time, avoid unnecessary jargon that would make your title impenetrable for anybody not working on the exact same question, but who would still find your research of interest or relevance to them. At Nature Human Behaviour, we sometimes suggest changes to the titles of the manuscripts we publish, to help them reach the widest possible audience.

Published online: 18 June 2021

https://doi.org/10.1038/s41562-021-01152-2

References

1. Subotić, S. \& Mukherjee, B. J. Inform. Sci. 40, 115-124 (2014).

2. Sagi, I. \& Yechiam, E. J. Inform. Sci. 34, 680-687 (2008). 